\title{
DURABILIDADE NATURAL DA MADEIRA DE DUAS ESPÉCIES PROVENIENTES DE FLORESTA ENERGÉTICA
}

\author{
Henrique Weber Dalla Costa ${ }^{1}$, Luana Candaten ${ }^{2}$, Rômulo Trevisan ${ }^{3}$, Darci Alberto \\ Gatto $^{4}$ \\ 1Doutorando do Programa de Pós-Graduação em Engenharia Florestal da Universidade \\ Federal de Santa Maria e-mail: hwdallacosta@gmail.com \\ ${ }^{2}$ Graduanda em Engenharia Florestal pela Universidade Federal de Santa Maria - Campus \\ Frederico Westphalen \\ ${ }^{3}$ Professor Doutor do Departamento de Engenharia Florestal da Universidade Federal de \\ Santa Maria - Campus Frederico Westphalen \\ ${ }^{4}$ Professor Doutor do Programa de Pós-Graduação em Engenharia Florestal da \\ Universidade Federal de Santa Maria
}

Recebido em: 22/09/2018 - Aprovado em: 23/11/2018 - Publicado em: 03/12/2018 DOI: 10.18677/EnciBio_2018B120

\begin{abstract}
RESUMO
A madeira contribui de múltiplas formas para a sociedade, entretanto, não apresenta elevada durabilidade natural quando exposta em ambientes que contribuem no desenvolvimento de organismos biológicos. Com isso, o estudo de suas propriedades nessas condições torna-se importante para compreender 0 comportamento do material em diferentes situações. A partir disso, o objetivo desse trabalho foi analisar a durabilidade natural da madeira de duas espécies provenientes de floresta energética. Para a condução do experimento foram confeccionados corpos de prova a partir de moirões de Mimosa scabrella e Acacia mearnsii. Esses passaram por exposição nas condições de floresta nativa, floresta plantada e campo para avaliação do comportamento do material e da característica química em cada uma das situações. A obtenção dos dados de propriedade química se deram a partir da norma ASTM D-1110. Os resultados evidenciaram que a solubilidade em água quente, de ambas espécies, decresceu em todos ambientes de exposição ao longo do tempo. Após 240 dias de exposição ocorreram os decréscimos mais acentuados. Na floresta plantada as espécies apresentaram comportamento semelhante, divergindo nas demais condições. A madeira de Mimosa scabrella apresentou maior durabilidade natural sendo menos deteriorada do que o material de Acacia mearnsii.
\end{abstract}

PALAVRAS-CHAVE: ensaio de campo, propriedade química. Solubilidade em água quente.

\section{NATURAL DURABILITY OF TWO WOOD SPECIES FROM ENERGY FORESTRY}

\begin{abstract}
The wood contributes to the society in many ways, however it does not have high natural durability when exposed in environments that further the development of biological organisms. Thus, the study of their properties under these conditions becomes important to understand the material's behavior in distinct situations. Therefore, the aim of this work was to analyze the natural durability of two wood
\end{abstract}


species from energy forestry. For experiment execution, stake specimens of Mimosa scabrella and Acacia mearnsii were made. The stakes were exposed in native forest, planted forest and meadow conditions to evaluate the material behavior and the chemical characteristic in each situation. The chemical property data were obtained from ASTM D-1110. The results shows both species hot-water solubility decreases along the time in all exposure condition. The most marked decrease occurred after 240 days of exposure. In planted forest both species showed same behavior, diverging in other conditions. The Mimosa scabrella wood showed larger natural durability, with less deterioration than Acacia mearnsii material.

KEYWORDS: hot-water solubility; chemical properties; field test.

\section{INTRODUÇÃO}

A madeira por suas propriedades particulares é um material que desde os primórdios colaborou no desenvolvimento das civilizações (TOMAZELLI et al., 2016). Apesar das características tecnológicas desejáveis, a mesma possui baixa durabilidade natural quando exposta em ambientes de elevada umidade (STALLBAUN et al., 2017). De maneira geral, todas as espécies florestais são susceptíveis ao ataque de organismos xilófagos, conforme Mattos et al. (2014), entretanto, a resistência a biodeterioração é uma propriedade altamente variável em função da composição química e anatômica do material (ROMANINI et al., 2014).

A durabilidade natural da madeira é interpretada pela capacidade que essa possui de resistir à ação de organismos xilófagos, sem nenhum tipo de tratamento preservativo (STALLBAUN, 2016). Essa característica pode ser determinada em ensaios de campo, embora métodos simples, reproduzem fielmente as condições reais de serviço nas quais as peças de madeira são expostas às intempéries e avaliadas quanto ao grau de deterioração, podendo a partir disso recomendar ou não o seu uso em ambientes externos (FOSCO MUCCI et al., 1992). Porém, quando o material é submetido a exposição a atmosfera, ocorre a chamada degradação fotoquímica, causando alterações nos polímeros, sendo esses, facilmente lixiviados pelas chuvas (MATTOS et al., 2014).

O comportamento da mesma madeira, segundo Cavalcante (1985), pode ser diferente em dois ambientes distintos, porque cada qual apresentará condições de, umidade relativa do ar, insolação, aeração, temperatura etc., que agem sobre o material, influenciando sua durabilidade. O estudo da deterioração da madeira, para Trevisan (2006) tem papel importante na otimização desse material, sendo relevante o completo entendimento desse processo, para um melhor e correto aproveitamento das propriedades de cada madeira, resultando dessa forma, na contenção de gastos e desperdícios, a exemplo, com reposição de peças.

Logo, torna-se importante determinar a durabilidade natural da madeira de diferentes espécies em ambientes distintos, a fim de prolongar a vida útil de seus subprodutos sem a necessidade do uso de tratamentos químicos. Dessa forma, o objetivo do trabalho foi analisar a durabilidade natural da madeira de duas espécies provenientes de floresta energética.

\section{MATERIAL E MÉTODOS}

O material utilizado para a condução do experimento foi coletado em uma floresta energética experimental, com cinco anos de idade, localizada na Universidade Federal de Santa Maria, Campus Frederico Westphalen. 
Foram abatidas 24 árvores de Mimosa scabrella (bracatinga) e Acacia mearnsii (acácia-negra). Destas, obteve-se 12 moirões de cada espécie com circunferência entre 20 e 30 centímetros e comprimento de 1,5 metro. Do topo de cada moirão seccionou-se um disco de $5 \mathrm{~cm}$ de altura, para a análise química da madeira pré-exposição. Os moirões, secos ao ar, foram instalados verticalmente com a base enterrada $40 \mathrm{~cm}$, com quatro repetições, nos três ambientes de exposição distintos, denominados de floresta nativa, floresta plantada e campo.

Durante o tempo de exposição, de 360 dias, foram coletados discos de cinco centímetros de espessura do topo de cada moirão a cada 120 dias. Os discos coletados foram secados em estufa a $103 \pm 2^{\circ} \mathrm{C}$ até massa constante, e retirada a casca. Após, foram transformados em serragem, utilizando-se moinho tipo Willey. Sequencialmente a serragem foi peneirada com auxílio de peneiras de 40 e 60 mesh, sendo utilizado o material retido na peneira de 60 mesh. A determinação do teor de umidade da madeira foi obtida utilizando alíquotas de $2 \mathrm{~g}$ do material, conforme a Equação 1:

$$
\mathrm{TU}=\frac{\mathrm{P}-\mathrm{Pas}}{\mathrm{P}} \mathrm{X} 100
$$

Onde: $\mathrm{TU}=$ Teor de umidade (\%); $\mathrm{P}=$ Massa inicial da amostra $(\mathrm{g}) ;$ Pas $=$ Massa da amostra absolutamente seca $(\mathrm{g})$.

O cálculo da porcentagem absolutamente seca foi realizado utilizando os valores observados no teste do teor de umidade (Equação 2), essa metodologia foi empregada para se obter alíquotas livres de qualquer umidade.

$$
\mathrm{AS}=\frac{\mathrm{Pas}}{\mathrm{P}} \times 100
$$

Onde: $\mathrm{AS}=$ Porcentagem absolutamente seca; $\mathrm{Pas}=$ Massa da amostra absolutamente seca $(\mathrm{g}) ; \mathrm{P}=$ Massa inicial da amostra $(\mathrm{g})$.

A determinação de extrativos pelo método da água quente seguiu os procedimentos propostos na norma ASTM D 1110-94 da American Society for Testing and Materials. Para a obtenção do percentual da amostra extraída em água quente (Eaq), foi utilizada a Equação 3:

$$
\mathrm{Eaq}=\frac{100^{*}(\mathrm{Pac}-\mathrm{PC})}{\mathrm{PaS}}-100
$$

Onde: $\mathrm{Eaq}=$ Teor de extrativos em água quente (\%); Pac = Massa da amostra + béquer de $250 \mathrm{~mL}$ (g); Pc = Massa do béquer de $250 \mathrm{~mL}(\mathrm{~g})$; Pas = Massa da amostra absolutamente seca (g).

Os resultados foram submetidos a análise de regressão para obtenção dos modelos matemáticos, utilizou-se o tempo como variável independente e a dependente foi a solubilidade em água quente, os critérios adotados para a seleção do melhor modelo foram: maior coeficiente de determinação ajustado ( $\left.R^{2} a j.\right)$, menor erro padrão de estimativa (Sxy) e maior valor de F calculado a significância de 1\%.

\section{RESULTADOS E DISCUSSÃO}

A partir dos dados observados foram gerados modelos matemáticos a fim de explicar a variação da solubilidade em água quente para a Acacia mearnsii e Mimosa scabrella, nos distintos ambientes de exposição. Os modelos que melhor descreveram esta variação estão apresentados na Tabela 1. 
TABELA 1 - Resumo estatístico dos modelos selecionados por espécie e ambiente de exposição.

\begin{tabular}{|c|c|c|c|c|c|c|c|c|}
\hline \multirow{2}{*}{$\begin{array}{c}\text { Ambiente de } \\
\text { exposição }\end{array}$} & \multirow{2}{*}{ Espécie } & \multirow{2}{*}{ Modelo } & \multicolumn{4}{|c|}{ Estatísticas } & \multirow{2}{*}{$-\mathbf{R}_{\text {aj. }}^{2}$} & \multirow[t]{2}{*}{ F } \\
\hline & & & $b_{j}$ & Valor & $F^{\prime}$ & Prob>F & & \\
\hline \multirow{3}{*}{ Campo } & & & $\begin{array}{l}b_{0} \\
b_{1}\end{array}$ & $\begin{array}{c}16,84 \\
-0,013\end{array}$ & $\begin{array}{c}1970,5 \\
58.32\end{array}$ & $\begin{array}{l}<0,001^{* *} \\
<0,001^{* *}\end{array}$ & 0,79 & 58,32 \\
\hline & Mim & Sol.H & $\mathrm{b}_{0}$ & 17,06 & 1461,5 & $<0,001^{* *}$ & & \\
\hline & & & $b_{1}$ & $-5,02 E^{-8}$ & 6 , & $1^{* *}$ & & \\
\hline \multirow{3}{*}{$\begin{array}{l}\text { Floresta } \\
\text { Nativa }\end{array}$} & & Sc & $b_{0}$ & 16,86 & 1460,8 & $<0,001^{* *}$ & \multirow{2}{*}{0,68} & \multirow{2}{*}{32,} \\
\hline & & & & $-0,011$ & & & & \\
\hline & $\begin{array}{l}\text { Mimosa } \\
\text { scabrella }\end{array}$ & $\begin{array}{l}\text { Sol. } \mathrm{H}_{2} \mathrm{O} \\
=\mathrm{b}_{0}+\mathrm{b}_{1} \mathrm{t}^{3}\end{array}$ & $\begin{array}{l}b_{0} \\
b_{1}\end{array}$ & $\begin{array}{c}16,40 \\
-5,23 E^{-8}\end{array}$ & $\begin{array}{c}6430,6 \\
39,51\end{array}$ & $\begin{array}{l}<0,001^{* *} \\
<0,001^{* *}\end{array}$ & 0,72 & 39, \\
\hline \multirow{2}{*}{$\begin{array}{l}\text { Floresta } \\
\text { plantada }\end{array}$} & $\begin{array}{c}\text { Acacia } \\
\text { mearnsii }\end{array}$ & $\begin{array}{l}\text { Sol. } \mathrm{H}_{2} \mathrm{O} \\
=b_{0}+b_{1} t\end{array}$ & $b_{0}$ & $\begin{array}{r}16,59 \\
-0.012\end{array}$ & $\begin{array}{l}1510,85 \\
40.87\end{array}$ & $\begin{array}{l}<0,001^{* *} \\
<0,001^{* *}\end{array}$ & 0,73 & 40,87 \\
\hline & $\begin{array}{c}\text { Mimosa } \\
\text { scabrella }\end{array}$ & $\begin{array}{l}\text { Sol. } \mathrm{H}_{2} \mathrm{O} \\
=\mathrm{b}_{0}+\mathrm{b}_{1} \mathrm{t}\end{array}$ & $\begin{array}{l}b_{0} \\
b_{1}\end{array}$ & $\begin{array}{r}16,79 \\
-0,009\end{array}$ & $\begin{array}{c}2836,0 \\
44,46\end{array}$ & $\begin{array}{l}<0,001^{* *} \\
<0,001^{* *}\end{array}$ & 0,14 & 44 \\
\hline
\end{tabular}

Onde: $\mathrm{Sol}_{\mathrm{H}} \mathrm{H}_{2} \mathrm{O}=$ Solubilidade em água quente, em \%; $\mathrm{b}_{\mathrm{j}}=$ coeficientes do modelo; $\mathrm{t}=$ tempo de exposição, em dias; F' = valor de $\mathrm{F}$ calculado para o modelo; Prob. $>\mathrm{F}=$ nível de probabilidade de erro; $R^{2}$ ai. = coeficiente de determinação ajustado; $F=$ valor de $F$ calculado; ${ }^{* *}=$ significativo ao nível de $1 \%$ de probabilidade de erro; ns = não significativo ao nível de $5 \%$ de probabilidade de erro.

A madeira de acácia-negra apresentou similaridade nas curvas de solubilidade em todos ambientes, de forma linear com decréscimo nos teores de compostos solúveis em água quente ao longo do tempo de exposição. Esta tendência, para a madeira de bracatinga, é válida apenas para a floresta plantada. Nos demais ambientes, há uma manutenção da solubilidade no início da exposição com posterior decréscimo a partir de 120 dias. No ambiente de campo (Figura 1) as espécies apresentaram distintas curvas de solubilidade, a madeira de acácia-negra apresenta diminuição desde o início da exposição, enquanto a madeira de bracatinga inicia entre 120 e 240 dias.

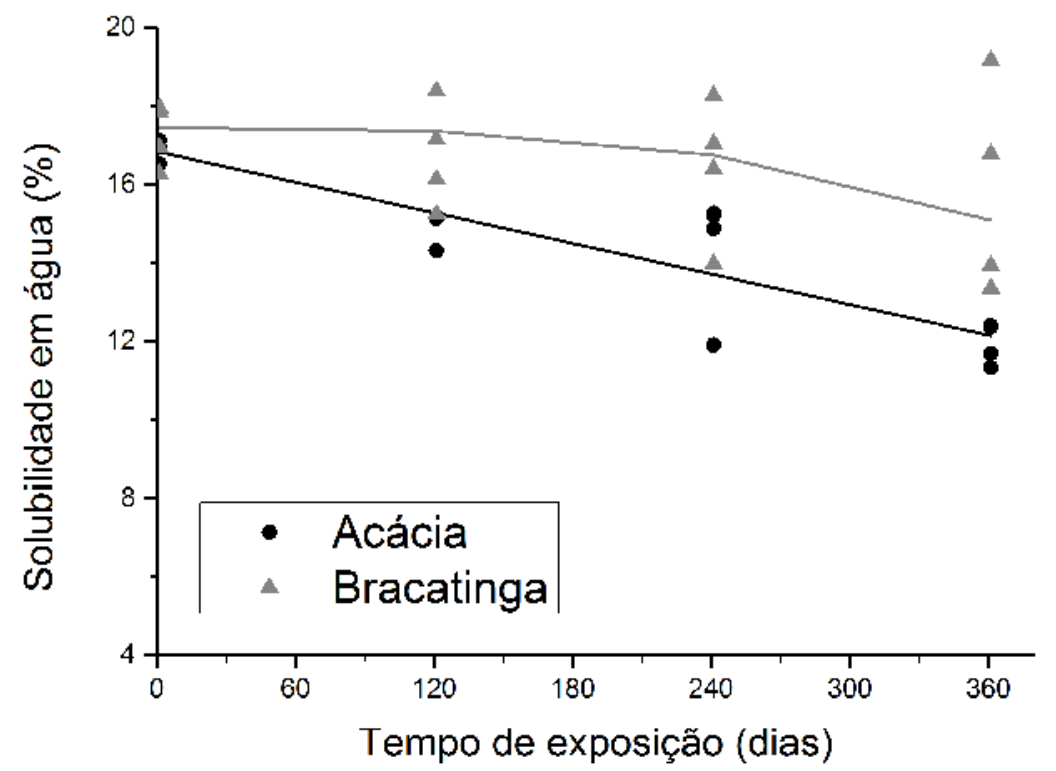

FIGURA 1 - Relação entre a solubilidade e o tempo de exposição no ambiente de campo. 
A solubilidade em água quente apresentou redução ao longo do tempo em ambas as espécies, sendo que essa ocorre de forma mais acentuada após o período 240 dias, no ambiente de floresta nativa (Figura 2).

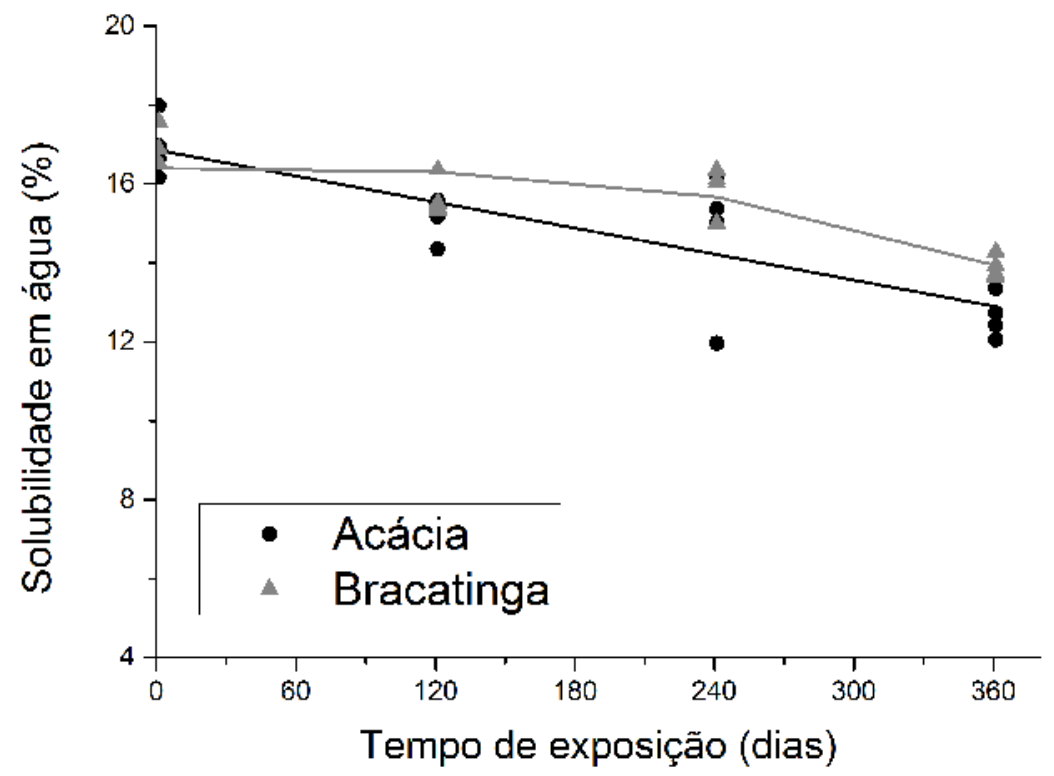

FIGURA 2 - Relação entre a solubilidade e o tempo de exposição no ambiente de floresta nativa.

No ambiente de floresta plantada (Figura 3) as duas espécies, acácia e bracatinga, demonstraram uma tendência de diminuição da solubilidade com o passar do tempo de exposição. Nesse ambiente, as espécies demonstraram o comportamento mais homogêneo, em comparação com a floresta nativa e com o campo.

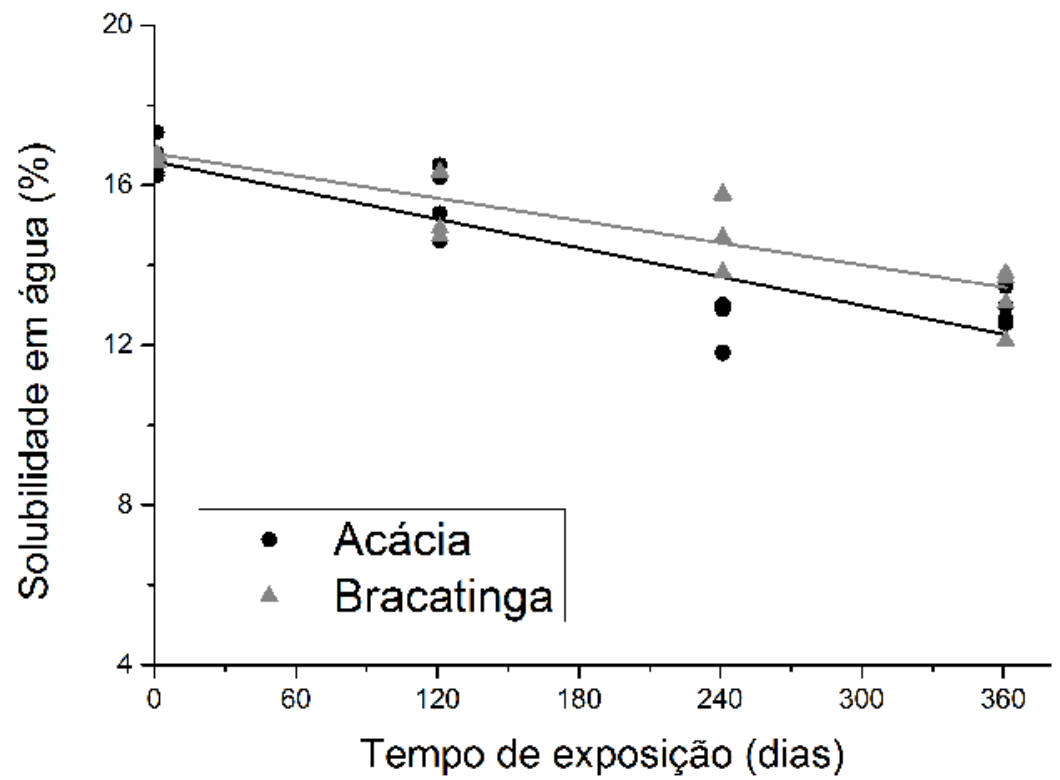

FIGURA 3 - Relação entre a solubilização e o tempo de exposição no ambiente de floresta plantada.

Esse comportamento observado mostra-se semelhante ao obtido por Mattos et al. (2014) que determinaram a variação de massa em estudo de durabilidade natural com diferentes espécies do gênero Eucalyptus, nas condições de campo e 
de floresta plantada. Os autores concluíram que ocorreu uma redução de massa ao longo do tempo, de forma análoga a solubilidade em água do presente estudo.

Em relação ao ambiente de exposição, para Mattos et al. (2014) a maior degradação ocorreu na floresta plantada, corroborando com o presente estudo. Entretanto, para Ribeiro et al. (2014) na floresta plantada houve uma menor perda de massa consequente da degradação. Os autores atribuíram essa tendência às características ecológicas do ambiente não florestal, que contribuíram no desenvolvimento de fungos e térmitas.

Entretanto, não somente por meio de organismos xilófagos que a madeira é degradada. Esta, por ser um material polimérico sofre alterações na sua composição, em função de reações fotoquímicas e térmicas provocadas pelo intemperismo, tais modificações acarretam na degradação da madeira (DE PAOLI, 2008). Portanto, a degradação na madeira entre os ambientes de exposição ocorreu de forma distinta em função das condições microclimáticas dos mesmos.

Em estudos com intemperismo, Yildiz et al. (2013), Tolvaj et al. (2014) e Baysal et al. (2014) atribuíram as maiores modificações em madeiras decorrentes da ação da radiação solar. A luz provoca a despolimerização da lignina, modificações nos extrativos e degradação das hemiceluloses em novos compostos químicos solúveis em água. Dessa forma, os novos compostos gerados são lixiviados da madeira pela água (chuva, orvalho, umidade ambiente), assim conferindo a umidade um importante fator na degradação (SILVA et al., 2007; TELES ; COSTA, 2014).

Para ambas as espécies, observou-se que ocorreu um decréscimo nos valores dos compostos solubilizados em água quente ao longo do tempo. A menor solubilização da madeira é resultado de processos de lixiviação e volatilização dos extraíveis para o meio, tendência também descrita por Brand e Muñiz (2012).

No ambiente de floresta plantada, ambas as espécies apresentaram constante decréscimo no teor de extrativos em água, caracterizado como ambiente em que ocorreu a maior degradação. A combinação de um ambiente com dossel em formação, que permite grande passagem de luz, com maior umidade conferida pela floresta em formação, favoreceu a lixiviação dos novos produtos formados pela modificação química causada na madeira.

Dessa forma, o ambiente mais indicado para uso da madeira das espécies em estudo seria a floresta nativa, pois foi onde se teve o menor grau de solubilização em água quente e com isso, menor degradação.

\section{CONCLUSÃO}

Os distintos microclimas dos ambientes de exposição influenciaram a durabilidade natural da madeira. A combinação do aumento da radiação solar (em comparação com a floresta nativa) e da umidade (em comparação com o campo) torna o ambiente de floresta plantada o mais favorável à degradação da madeira.

O ambiente de floresta plantada foi o que teve menos influência no comportamento das espécies em estudo em relação a degradação. De maneira geral, a madeira de Mimosa scabrella é a mais recomendada para uso em ambientes externos em função de sua maior resistência natural quando comparada com a Acacia mearnsii.

A degradação da madeira gera constituintes hidrossolúveis que são lixiviados pela umidade do ambiente e, ou pelas chuvas, portanto, quanto menor o teor de extrativos solúveis em água maior a degradação do material no campo, ou seja, há uma correlação direta entre solubilidade em água e durabilidade natural da madeira. 


\section{AGRADECIMENTOS}

O presente trabalho foi realizado com apoio do Fundo de Incentivo à Pesquisa por meio do Programa FIPE Júnior/UFSM.

\section{REFERÊNCIAS}

AMERICAN SOCIETY FOR TESTING AND MATERIALS - ASTM. (ASTM D 1110). Standard test method for water solubility of wood. Annual Book of ASTM standards: wood. Philadelphia: 1994. v. 0410, p. 195-196.

BAYSAL, E.; KART, S.; TOKER, H.; DEGIRMENTEPE, S. Some physical characteristics of thermally modified oriental-beech wood. Maderas. Ciencia y tecnología, v. 16, n. 3, p. 291-298, 2014. Disponível em: < http://revistas.ubiobio.cl/index.php/MCT/article/view/1164>. doi: 10.4067/S0718$221 \times 2014005000022$

BRAND, M. A.; MUÑIZ, G. I. B. de; Influência da Época de Colheita e da Estocagemna Composição Química da Biomassa Florestal. Floresta e Ambiente, Rio de Janeiro, v.19, n. 1, p. 66-78, 2012. Disponível em: < https://www.researchgate.net/publication/276006210_Influencia_da_Epoca_de_Colh eita_e_da_Estocagem_na_Composicao_Quimica_da_Biomassa_Florestal>. doi: 10.4322/floram.2012.008.

CAVALCANTE, M. S. Métodos para aumentar a durabilidade da madeira. Boletim da Associação Brasileira de Preservadores de Madeira. São Paulo, n 36, p 159170, 1985

DE PAOLI, M. A. Degradação e Estabilização de Polímeros. São Paulo: Artliber Editora, 2008. 286.

FOSCO MUCCI, E. S. C.; LOPEZ, G. A.; MONTAGNA, R. G. Durabilidade natural de madeiras em contato com o solo - IV. Revista do Instituto Florestal. São Paulo, v.4, parte 2, p. 558-563, 1992.

MATTOS, B. D.; MISSIO, A. L.; GATTO, D. A. Resistência natural da madeira de três espécies de eucaliptos submetidas a dois sítios. XX Congresso de Iniciação Científica, III Mostra Científica, 2014.

RIBEIRO, M. A.; STANGERLIN, D. M.; SOUZA, A. P. de; CARDOSO, G. V.; CALEGARI, L. et al. Durabilidade natural da madeira de jequitibá em ensaios de deterioração em campo aberto e floresta durante as estações de seca e chuva. Comunicata Scientiae, Piauí, v. 5, n. 4, p. 402-411, 2014. Disponível em: < https://www.comunicatascientiae.com.br/comunicata/article/view/262/279>.

ROMANINI, A.; STANGERLIN, D. M.; PARIZ, E.; SOUZA, A. P. de; GATTO, D. A.; CALEGARI, L. Durabilidade natural da madeira de quatro espécies amazônicas em ensaios de deterioração de campo. Nativa, Sinop, v. 02, n. 01, p. 13-17, 2014. Disponível em: <http://periodicoscientificos.ufmt.br/ojs/index.php/nativa/article/ view/1309>. doi: 10.14583/2318-7670.v02n01a03 
SILVA, J. O. E.; PASTORE, T. C. M.; JUNIOR, F. P. Resistência ao intemperismo artificial de cinco madeiras tropicais e de dois produtos de acabamento. Ciência Florestal, v. 17, n. 1, p. 7, 2007. Disponível em: < https://periodicos.ufsm.br/cienciaflorestal/article/view/1931/1173>.

STALlBAUn, P. H.; BARAUnA, E. E. P.; MONTEIRO, T. C.; VIEIRA, R. da S.; SALES, N. de L. P. et al. Resistência natural da madeira de Tachigali vulgaris ao fungo xilófago Postia placenta. Pesquisa Florestal Brasileira, Colombo, v. 36, n. 88, p. 2016. 459-463, Disponível em: < https://pfb.cnpf.embrapa.br/pfb/index.php/pfb/article/view/1231/539>. doi: 10.4336/2016.pfb.36.88.1231.

STALLBAUN, P. H.; BARAUNA, E. E. P.; PAES, J. B.; RIBEIRO, N. C.; MONTEIRO, T. C.; ARANTES, M. D. C. Resistência Natural da Madeira de Sclerolobium paniculatum Vogel a Cupins em Condições de Laboratório. Floresta e Ambiente, Seropédica, v. 24, 2017. Disponível em: <http://www.scielo.br/pdf/floram/v24/21798087-floram-2179-8087001316.pdf>. doi: 10.1590/2179-8087.001316.

TELES, R. F.; COSTA, A. F. Influência do Intemperismo Acelerado nas Propriedades Colorimétricas da Madeira de Angelim Pedra. Revista Nativa, v. 2, n. 2, p. 5, 2014. Disponível em: < http://periodicoscientificos.ufmt.br/ojs/index.php/nativa/article/view/ 1388>. doi: 10.14583/2318-7670.v02n02a02

TOMAZELI, A. J.; SILVEIRA, A. G. da; TREVISAN, R.; WASTOWSKI, A. D.; CARDOSO, G. V. Durabilidade natural de quatro espécies florestais em campo de apodrecimento. Tecno-lógica, Santa Cruz do Sul, v. 20, n. 1, p. 20-25, 2016. Disponível em: <https://online.unisc.br/seer/index.php/tecnologica/article/view/6473>. doi: 10.17058/tecnolog.v20i1.6473.

TOLVAJ, L.; NEMETH, R.; PASZTORY, Z.; BEJO, L.; TAKATS, P. Colour Stability of Thermally Modified Wood during Short-Term Photodegradation. 2014. ISBN 1930-2126.

TREVISAN, H. Degradação natural de toras e sua influência nas propriedades físicas e mecânicas da madeira de cindo espécies florestais. 2006. 56 f. Dissertação (Mestrado em Ciências Ambientais e Florestais) - Universidade Federal Rural do Rio de Janeiro, Seropédica, RJ, 2006.

YILDIZ, S.; TOMAK, E. D.; YILDIZ, U. C.; USTAOMER, D. Effect of artificial weathering on the properties of heat treated wood. Polymer Degradation and Stability, v. 98, n. 8, p. 1419-1427, 2013. Disponível em: < https://www.sciencedirect.com/science/article/pii/S0141391013001328>. doi: 10.1016/j.polymdegradstab.2013.05.004 